\title{
Neuralgia do trigêmeo periférica tratada com alcoolização: relato de caso
}

Peripheral trigeminal neuralgia treated with alcoholization: a case report

Neuralgia del trigémino periférico tratada con alcoholización: reporte de caso

\section{Lucas Berlatto MODONESI}

Leandro da Cunha DIAS ${ }^{2}$

Daniele Lacerda PEREIRA ${ }^{3}$

Adriana Haack de Arruda DUTRA ${ }^{4}$

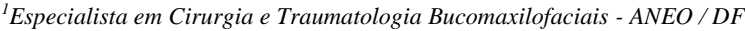

Residente em Cirurgia e Traumatologia Bucomaxilofaciais, Associação Nacional de Estudos Odontológicos ANEO, Mestrando em Ciências para a Saúde - ESCS /DF Especialista em Cirurgia e Traumatologia Bucomaxilofaciais - ANEO / DF

${ }^{3}$ Residente em Cirurgia e Traumatologia Bucomaxilofaciais, Associação Nacional de Estudos Odontológicos ANEO,

Especialista em Cirurgia e Traumatologia Bucomaxilofaciais - ANEO / DF

${ }^{4}$ Mestre em Nutrição Humana - UnB, Doutora em Ciências da Saúde - UnB, Docente titular do Programa de Pós-Graduação em Ciências para a Saúde - ESCS / DF

\section{Resumo}

Os casos de neuralgia do trigêmeo no âmbito da Odontologia merecem, ainda, maior atenção devido ao difícil e criterioso diagnóstico desta condição que, muitas vezes, acaba confundida com outras patologias associadas às dores orofaciais. Este trabalho visa relatar o caso de uma paciente idosa edêntula com quadros de dor em topografia de nervo mentual direito, com sensibilização em mucosa gengival e lábio inferior à direita, compatível com neuralgia trigeminal, a qual foi tratada com indução à neurólise do nervo mentual direito por necrose asséptica estimulada por solução alcoólica. A paciente encontra-se com dois anos de acompanhamento e sem os episódios de dor. Mesmo o processo de diagnóstico sendo extenso e exigindo muito critério, foi possível diagnosticar a nevralgia da paciente e empregar o passo-a-passo do tratamento, chegando a melhor opção para este caso, que foi a alcoolização do nervo mentual.

Descritores: Neuralgia Facial; Neuralgia; Neuralgia do Trigêmeo; Bloqueio Nervoso.

\section{Abstract}

The cases of trigeminal neuralgia in the scope of Dentistry deserve even more attention due to the difficult and criterious diagnosis of this condition, which often ends up confused with other pathologies associated with orofacial pains. This study aims to report the case of an elderly edentulous patient with pain episodes on the right mental nerve's topography, with sensitivity on the gingival mucosa and lower right lip, compatible with trigeminal neuralgia, which was treated with induction to neurolysis of the right mental nerve by aseptic necrosis stimulated by alcoholic solution. The patient has two years of follow-up whithout pain's episodes. Even though the diagnostic process was extensive and required a lot of criteria, it was possible to diagnose the patient's neuralgia and to use step-by-step treatment, arriving at the best option for this case, which was the mental nerve's alcoholization.

Descriptors: Facial Neuralgia; Neuralgia; Trigeminal Neuralgia; Nerve Block.

\section{Resumen}

Los casos de neuralgia del trigémino en el ámbito de la Odontología merecen, aún, mayor atención debido al difícil y criterioso diagnóstico de esta condición que, muchas veces, acaba confundida con otras patologías asociadas a los dolores orofaciales. Este trabajo trata de relatar el caso de una paciente anciana edéntula con cuadros de dolor en topografía de nervio mental derecho, con sensibilización en mucosa gingival y labio inferior a la derecha, compatible con neuralgia trigeminal, la cual fue tratada con inducción a la neurólisis del nervio mental derecho por necrosis aséptica estimulada por solución alcohólica. La paciente se encuentra con dos años de seguimiento y sin los episodios de dolor. Incluso el proceso de diagnóstico siendo extenso y exigiendo mucho criterio, fue posible diagnosticar la neuralgia de la paciente y emplear el paso a paso del tratamiento, llegando la mejor opción para este caso, que fue la alcoolización del nervio mental.

Descriptores: Neuralgia Facial; Neuralgia; Neuralgia del Trigémino; Bloqueo Nervioso.

\section{INTRODUÇÃO}

As neuralgias são síndromes álgicas restritas a área de inervação de um determinado nervo. A mais comum na face é a neuralgia do trigêmeo (NT), sendo, também, uma das condições dolorosas mais conhecidas pelos humanos ${ }^{1}$.

A neuralgia trigeminal é uma condição associada ao nervo trigêmeo, sendo caracterizada por intenso quadro álgico no território de inervação dos ramos desse nervo. $\mathrm{O}$ nervo trigêmeo, classificado como o quinto par craniano, é o principal nervo sensitivo da cabeça e também inerva a musculatura da mandíbula. É considerado um nervo misto, por possuir uma raiz motora e uma raiz sensitiva, embora seja predominantemente sensitivo. Possui fibras aferentes e eferentes, e subdivide-se em três ramos, os quais em ordem descendente são, respectivamente: nervo oftálmico (V1), nervo maxilar (V2) e nervo mandibular (V3). Os neurônios sensitivos formam o nervo maxilar, nervo oftálmico e nervo mandibular, através das fibras aferentes somáticas gerais, e são responsáveis pela sensibilidade somática geral da maior parte da cabeça. Estas fibras conduzem impulsos exteroceptivos e impulsos proprioceptivos.
Sua parte motora inerva os músculos mastigatórios masseter e pterigoideos ${ }^{2}$.

A International Headache Society (IHS) define a NT como "uma afecção caracterizada por uma dor recorrente, espasmódica, unilateral, tipo choque elétrico, breve, de início e fim abruptos, e limitada ao território de distribuição de uma ou mais divisões do nervo trigêmeo e despertada por estímulos inócuos na região dos pontos de gatilho (trigger points). A duração de uma única dor espasmódica é inferior a dois minutos, embora o ataque total possa consistir em numerosos espasmos de curta duração. A dor é comumente evocada por estímulos triviais, toques suaves ou estímulos termais, onde podem ser incluídos os atos de lavar a face, barbear, maquiar, fumar, mascar, engolir, escovar os dentes e expor-se ao vento; entretanto, pode também ocorrer espontaneamente. Entre os ataques de dores paroxismais, o paciente é, geralmente, livre de dor, mesmo estimulando os pontos de gatilho ${ }^{3}$.

A incidência da NT é maior nos pacientes acima de 40 anos, ligeiramente mais incidente entre 
as mulheres, sendo o lado direito mais comumente afetado. Qualquer ramo do trigêmeo pode ser afetado, estando o oftálmico em apenas 5\% dos $\operatorname{casos}^{2,3}$.

A proximidade dos dentes com a localização da dor é comum nessa neuropatia, podendo sugerir, erroneamente, uma causa odontogênica para a dor. Devido a isso, é comum o cirurgião-dentista ser o primeiro profissional a ter contato com o paciente ${ }^{4}$. Por isso, é imprescindível que o cirurgião-dentista esteja atento para a realização de seu diagnóstico diferencial, a fim de evitar a realização de procedimentos odontológicos desnecessários nos pacientes, causando maior sofrimento e transtornos aos mesmos ${ }^{5}$.

A IHS distingue entre a NT clássica e a sintomática. A clássica inclui todos os casos sem etiologia estabelecida (idiopática) e requer que não exista evidência clínica de déficit neurológico e nem anormalidades laboratoriais ou radiográficas que justifiquem os episódios de dor. Já o diagnóstico da NT sintomática é feito quando a investigação identifica outra anormalidade estrutural, além da compressão vascular, que está afetando o nervo trigeminal. Estas anormalidades incluem esclerose múltipla, tumores e anormalidades da base do crânio ${ }^{3}$.

O presente trabalho tem por objetivo relatar os critérios diagnósticos utilizados para identificar a presença de neuralgia do trigêmeo clássica em uma paciente idosa, bem como as opções de tratamento levadas em consideração e o tratamento que foi resolutivo neste caso (a alcoolização), ilustrando a importância do conhecimento e manejo dessa enfermidade pelos cirurgiões-dentistas.

\section{CASO CLÍNICO}

Paciente de 65 anos de idade, sexo feminino, moradora de asilo, procurou nosso serviço de Cirurgia e Traumatologia Bucomaxilofacial na cidade de Brasília - DF, queixando-se de dificuldades para se alimentar devido a dores na arcada inferior, dores essas que se manifestavam independente do alimento estar frio ou quente; e relatava, também, dor com estímulos leves de toque. Apresentava-se, também, com aparência prostrada e pouco comunicativa.

Segundo a acompanhante, que é cuidadora do asilo, a paciente em questão costumava ser muito animada e comunicativa antes do aparecimento dos sintomas. Ressaltou que a paciente já vinha se alimentando, há algum tempo, apenas com dieta líquido-pastosa, o que a debilitou ainda mais ("ela já estava evitando se alimentar, pois não conseguia comer as coisas que gostava pela dor").

Após colher todas as informações referentes à história da moléstia atual da paciente, buscou-se obter informações detalhadas sobre seu estado geral de saúde e possíveis comorbidades que pudessem estar associadas à dor, chegando-se à conclusão de que se tratava de uma paciente idosa sadia, sem alterações locais ou sistêmicas que justificassem a dor relatada.

No exame físico, notou-se que a paciente era edêntula e fazia uso de prótese total inferior e superior, ambas bem adaptadas, sem sinais de traumatismos em rebordos alveolares e/ou mucosa bucal. Além disso, pontos-gatilho foram identificados em lábio inferior à direita e sobre a mucosa gengival próxima a região de pré-molares e de segundo molar, também à direita.

Foi solicitada radiografia panorâmica, a fim de se investigar possíveis alterações não visíveis clinicamente, a qual não evidenciou anormalidades, podendo ser excluídas as manifestações clínicas de origem odontogênica ou de formação dos tecidos da face. Assim, incluiu-se como hipótese diagnóstica a neuralgia do trigêmeo, e passou-se a investigar tal condição. Para tanto, usou-se os critérios diagnósticos de NT da International Headache Society estipulados no manual de Classificação Internacional de Cefaleias (versão ICHD-3 Beta) ${ }^{6}$, o qual estabelecia os seguintes critérios:

A) Pelo menos três episódios de dor facial unilateral preenchendo os critérios $B$ e $C$;

$B$ ) Ocorrendo em uma ou mais divisões do nervo trigêmeo e sem irradiação para além da distribuição do trigêmeo;

C) A dor apresenta, pelo menos, três das seguintes quatro características:

$>$ recorrente em acesso paroxístico, durando de uma fração de segundo a 2 minutos;

$>$ intensidade grave;

$>$ tipo choque elétrico, fisgada, facada ou guinada;

$>$ desencadeada por estímulos inócuos no lado afetado da face;

D) Não há déficit neurológico clinicamente evidente.

Após concluir a avaliação, verificou-se que os sintomas da paciente eram compatíveis com as opções "A, B, C1, C2, C3, C4 e D". Com isso, ficou estabelecido o diagnóstico de neuralgia trigeminal clássica, por não incluir nos achados esclerose múltipla, tumores e anormalidades da base do crânio. Conforme preconizado em literatura, iniciou-se o tratamento com uso de medicação antiepiléptica.

$\mathrm{Na}$ primeira semana foi instituído o uso de carbamazepina 200mg de $12 \mathrm{em} 12$ horas, sendo a paciente reavaliada com sete dias de tratamento. Ao retornar, verificou-se a ineficácia do medicamento, e então o intervalo entre as doses foi diminuído para oito em oito horas. No segundo retorno, já com 14 dias de tratamento, a paciente relatou pequena melhora nos episódios de dor, e a mesma encontravase um pouco mais comunicativa, mas nada que a possibilitasse, ainda, ter uma vida normal. Optou-se, então, em diminuir o intervalo das doses para uma dose a cada seis horas. Na terceira semana de uso da 
medicação em dose máxima, a paciente não relatou melhora comparada a segunda semana, e queixava-se de muita letargia, decorrente da ação do medicamento a nível central. A paciente desejou não seguir com o tratamento, pois, segundo ela, houve nova queda em sua qualidade de vida, uma vez que ainda sentia as dores e ficava letárgica. Ela foi, então, aconselhada a manter a medicação por mais uma semana, na tentativa de não ser submetida a procedimento cirúrgico e também pelo fato de que os anticonvulsivantes não são medicamentos analgésicos e dependem do tempo para ter alguma eficácia, sendo esta atingida frequentemente entre 20 e 30 dias de tratamento.

Em novo retorno, já com quatro semanas de uso do medicamento, a paciente insistiu que não houve melhora em sua sintomatologia dolorosa comparada a segunda semana, e a letargia ainda persistia. Sendo assim, o uso da carbamazepina foi suspenso e escolheu-se realizar o procedimento menos invasivo possível antes de um procedimento cirúrgico radical, que incluiria a neurectomia do nervo mentual.

O tratamento de escolha foi alcoolização do nervo mentual. A técnica consistiu em antissepsia do campo operatório e aposição dos campos cirúrgicos estéreis, bloqueio do nervo alveolar inferior direito com solução anestésica de mepivacaína $2 \%$ com adrenalina 1:100.000, punção cutânea com seringa hipodérmica para acesso ao forame mentual direito e infiltração de $2 \mathrm{~mL}$ de solução de álcool a $70 \%$, sob pressão. É importante verificar que se está no interior do forame mentual para que a infiltração entre em contato apenas com o nervo, evitando extravasamento para os tecidos adjacentes, bem como realizar aspiração prévia a fim de se precaver de uma injeção intravascular.

Foram necessárias três infiltrações de $2 \mathrm{~mL}$ da solução alcoólica a $70 \%$, seguindo a técnica descrita e com intervalo de sete dias entre cada aplicação, para resolução do quadro doloroso da paciente. Por fim, a mesma relatou satisfação com o resultado alcançado e logrou mais qualidade de vida, retornando às suas atividades de vida diária.

\section{DISCUSSÃO}

A neuralgia do trigêmeo é responsável por uma das dores mais severas e intensas que uma pessoa pode vir a experimentar em sua vida ${ }^{7,8}$. Apresenta um quadro clínico típico e característico, praticamente patognomônico ${ }^{9}$. É uma algia da face severa, de início agudo, onde as áreas de dor intensa são aquelas de distribuição das divisões do nervo trigêmeo ${ }^{1}$.

Pacientes que apresentam dor orofacial podem, eventualmente, procurar o primeiro atendimento com cirurgiões-dentistas. Segundo Bertoli $^{5}$, quando pacientes procuram clínicas odontológicas com queixas de dor, a primeira hipótese diagnóstica é, na maioria dos casos, dor odontogênica. Devido a isso, é fundamental que o cirurgião-dentista saiba, ao menos, identificar uma possível NT e encaminhar o paciente ao profissional mais capacitado a tratá-lo 5 .

Conforme descreve a literatura ${ }^{2}$, a NT é mais incidente em mulheres acima dos 40 anos de idade e afeta mais comumente o lado direito, o que é corroborado neste estudo. Dentro dos tratamentos preconizados, a terapia medicamentosa é considerada o tratamento de primeira linha para a NT. O anticonvulsivante carbamazepina tem sido largamente usado desde 1960 devido a sua eficácia em aproximadamente $60 \%$ a $80 \%$ dos pacientes ${ }^{10}$, motivo pelo qual foi a primeira opção terapêutica para o presente caso.

Outros autores também reforçam que a primeira opção terapêutica deve ser não invasiva, através de fármacos anticonvulsivantes e/ou antidepressivos; entre os principais ressaltam, também, a carbamazepina, que atua bloqueando canais de sódio voltagem-dependentes pela redução dos disparos repetitivos de alta frequência do potencial de ação, impedindo a condução de impulsos nervosos aferentes e bloqueando a deflagração do quadro doloroso ${ }^{11-14}$.

O tratamento cirúrgico é indicado quando a terapia farmacológica falha, o paciente não consegue adaptar-se aos efeitos colaterais ou se alguma lesão central é diagnosticada ${ }^{15}$. Ratificando isso, em nosso estudo aqui presente, houve tanto falha do tratamento farmacológico instituído quanto não adaptação por parte da paciente aos efeitos colaterais causados pelo mesmo.

São várias as técnicas cirúrgicas para tratamento da neuralgia trigeminal em caso de falência do tratamento medicamentoso, sendo descritas por inúmeros autores. Segundo Quesada et al. ${ }^{4}$, entre essas técnicas há a neurólise do nervo maxilar e do nervo mandibular, neurólise nos forames supraorbitário, infraorbitário e canal mandibular, neuro-exérese dos ramos terminais, secção dos ramos do trigêmeo na base do crânio, infiltração alcoólica, eletrocoagulação, gasserrectomia, rizotomia retroglasseriana e termocoagulação do gânglio de Gasser por radiofreqüência ${ }^{4}$. Optou-se inicialmente, neste estudo, por uma técnica cirúrgica menos invasiva - a alcoolização - cujo objetivo era a neurólise do nervo associado à neuralgia (nervo mentual) através de uma necrose asséptica estimulada pela infiltração da solução alcoólica.

\section{CONSIDERACÕES FINAIS}

É de fundamental importância o conhecimento da neuralgia do trigêmeo pelo cirurgião-dentista, visto que, por se tratar de uma dor 
orofacial com aspectos intimamente relacionados com a cavidade oral, esse profissional muitas vezes será o de primeiro contato. Isso não implica que o mesmo saiba tratar a condição, entretanto, é fundamental que saiba incluí-la em seu diagnóstico diferencial, para evitar maiores perdas/transtornos ao paciente, e encaminhá-lo a um profissional capacitado a realizar o tratamento.

Também é notório que a alcoolização do feixe nervoso envolvido na neuralgia é válida e eficaz, sendo um dos tratamentos invasivos mais básicos a ser realizado, porém, com grande potencial de resolução do quadro álgico. Deve-se ressaltar que, entretanto, quando a afecção se dá a nível troncular ou no gânglio trigeminal, o tratamento foge do escopo do cirurgião-dentista, devendo ser instituído exclusivamente pelo médico.

\section{REFERÊNCIAS}

1. Siqueira SRDT, Siqueira JTT. Neuralgia do trigêmeo: diagnóstico diferencial com odontalgias. APCD. 2003.

2. Leocádio JCM, Santos LC, Sousa MCA, Gonçalves NJC, Campos IC. Neuralgia do trigêmeo: uma revisão de literatura. Braz J Surg Clin Res. 2014;7(2):33-7.

3. Headache Classification Subcommitte of the International Headache Society. The International Classification of Headache Disorders. 2nd ed. Cephalalgia. 2004;24(Suppl):9-160.

4. Quesada GAT, Baptista CE, Pedroso DS, Flores DL. Neuralgia trigeminal: do diagnóstico ao tratamento. Rev Dentíst Online. 2005;5(11):46-54.

5. Bertoli FMP, Koczicki VC, Meneses MS. A neuralgia do trigêmeo: um enfoque odontológico. JBA. 2003;3(10):125-29.

6. Classificação Internacional de Cefaleias. 3. ed. 2014. Disponível em: https://www.ichd-3.org/wpcontent/uploads/2016/08/2087_ichd-3-betaversao-pt-portuguese.pdf. Acesso em 04 out 2018.

7. Domingues RB, Kuster GW, Aquino $\mathrm{CCH}$. Treatment of trigeminal neuralgia with low doses of topiramate. Arq Neuropsiquiatr. 2007; 65(3B):792-94.

8. Borbolato RM, Ambiel CR. Neuralgia do trigêmeo: aspectos importantes na clínica odontológica. Saúde e Pesquisa. 2009;2(2):201-8.

9. Mattos JMB, Bueno FV, Mattos LR. Neuralgia do trigêmeo: um novo protocolo de tratamento clínico. Rev Dor. 2005;6(4):652-56.

10.Kitt CA, Gruber K, Davis M, Woolf CJ, Levine JD. Trigeminal neuralgia: opportunities for research and treatment. Pain. 2000;85(1-2):3-7.

11.Frizzo HM, Hasse PN, Veronese RM. Neuralgia do trigêmeo: revisão bibliográfica analítica. Rev Cir Traumatol Buco-Maxilo-Facial. 2004; 4(4):212-17.
12.Alves TCA, Azevedo GS, Carvalho ES. Tratamento farmacológico da neuralgia do trigêmeo: revisão sistemática e metanálise. Rev Bras Anestesiol. 2004;54(6):836-49.

13.Felix F, Olivaes MCD, Gismondi RAOC, Belmont H, Felix JAP. Tratamento conservador da síndrome de Gradenigo. Rev Bras Otorrinol. 2003;69(2):256-59.

14.Okeson JP. Dores bucofaciais de Bell: tratamento clínico da dor bucofacial. 6. ed. São Paulo: Quintessence, 2006.

15.Poluha RL, Silva RS. Neuralgia do trigêmeo V3: relato de caso. Rev Uningá. 2015;45(1):40-2.

\section{CONFLITO DE INTERESSES}

Os autores declaram não haver conflitos de interesse.

\section{AUTOR PARA CORRESPONDENCIA}

\section{Leandro da Cunha Dias}

leandrocdias@yahoo.com.br

Submetido em 14/10/2018

Aceito em 12/03/2019 